\title{
RILIS-ionized mercury and tellurium beams at ISOLDE CERN
}

\author{
T. Day Goodacre ${ }^{1,2} \cdot$ J. Billowes ${ }^{2} \cdot$ K. Chrysalidis ${ }^{1,3}$ • D. V. Fedorov ${ }^{4}$ \\ V. N. Fedosseev ${ }^{1}$ - B. A. Marsh' ${ }^{1}$ - P. L. Molkanov ${ }^{4}$ R. E. Rossel ${ }^{1,3,5}$. \\ S. Rothe $\mathrm{R}^{1,2}$. C. Seiffert ${ }^{1}$. K. D. A. Wendt ${ }^{3}$
}

Published online: 22 February 2017

(C) The Author(s) 2017. This article is published with open access at Springerlink.com

\begin{abstract}
This paper presents the results of ionization scheme development for application at the ISOLDE Resonance Ionization Laser Ion Source (RILIS). Two new ionization schemes for mercury are presented: a three-step three-resonance ionization scheme, ionizing via an excitation to a Rydberg level and a three-step two-resonance ionization scheme, with a non-resonant final step to the ionization continuum that corresponded to a factor of four higher ionization efficiency. The efficiency of the optimal mercury ionization scheme was measured, together with the efficiency of a new three-step three resonance ionization scheme for tellurium. The efficiencies of the mercury and tellurium ionization schemes were determined to be $6 \%$ and $>18 \%$ respectively.
\end{abstract}

Keywords RILIS · ISOLDE · Mercury · Tellurium · Laser ion source

This article is part of the Topical Collection on Proceedings of the 10th International Workshop on Application of Lasers and Storage Devices in Atomic Nuclei Research: "Recent Achievements and Future Prospects” (LASER 2016), Poznań, Poland, 16-19 May 2016

Edited by Krassimira Marinova, Magdalena Kowalska and Zdzislaw Błaszczak

T. Day Goodacre

thomas.day.goodacre@cern.ch

1 CERN, 1211 Geneva 23, Switzerland

2 School of Physics and Astronomy, The University of Manchester, Manchester, M13 9PL, UK

3 Institut für Physik, Johannes Gutenberg Universität, 55099 Mainz, Germany

4 Petersburg Nuclear Physics Institute, 188350 Gatchina, Russia

5 Faculty of Design, Computer Science and Media, Hochschule RheinMain, Wiesbaden, Germany 


\section{Introduction}

The Resonance Ionization Laser Ion Source (RILIS) [1, 2] is the principal ion source of the ISOLDE radioactive ion beam facility [3] based at CERN, scheduled for $>75 \%$ of experiments in 2016. The RILIS targets sequential atomic resonances to achieve the step-wise excitation of a valence electron, followed by a final transition resulting in ionization. The final step transition is either an excitation to an autoionizing state, a Rydberg level or a non-resonant transition to the ionization continuum. The sequential series of atomic excitations is termed the "ionization scheme" of the element. The primary advantage of the RILIS, with respect to the other ionization mechanisms employed at ISOLDE, is the element selective nature of the ionization process. This is crucial, when the production of isobaric contaminants can be orders of magnitude higher than that of the isotope of interest. The prerequisite for applying the RILIS to a particular element is that a multi-step ionization scheme must be developed. Following the determination the ionization scheme corresponding to the highest ion signal, the absolute efficiency of the ionization scheme is measured. This enables an assessment of the suitability of the ionization scheme for application at ISOLDE.

\subsection{Mercury}

A pre-existing ionization scheme for mercury, was based on a publication by A.A. Podshivalov et al. [4]. It was determined to have an ionization efficiency of $0.1 \%$ when tested at the ISOLDE RILIS [5]. The three step $\left(\left\{\lambda_{1}\left|\lambda_{2}\right| \lambda_{3}\right\}=\{254 \mathrm{~nm}|313 \mathrm{~nm}| 626 \mathrm{~nm}\}\right)$ ionization scheme from [4] uses the residual $626 \mathrm{~nm}$ fundamental light from the $2 \omega$ conversion process (producing the $313 \mathrm{~nm}$ second step) as the final ionizing transition. It was suggested that the $626 \mathrm{~nm}$ light accessed a favourable part of the ionization continuum due to the vicinity of autoionizing states. By comparison, the reported ionization efficiency of the FEBIAD type ion source for mercury is $60 \%$ [6]. There was therefore limited interest for the application of the RILIS scheme on-line at ISOLDE. A more efficient ionization scheme was required to facilitate an in-source resonance ionization spectroscopy experiment of mercury isotopes, seeking to extend the optical measurements of the mercury isotope chain further into the neutron rich and neutron deficient regions of the nuclear chart [7]. The spectroscopic investigations that resulted in a new mercury ionization scheme are presented in Section 3.1 and the efficiency measurement of this ionization scheme is presented in Section 3.2.

\subsection{Tellurium}

A RILIS scheme for tellurium was requested to enable signal identification for a Coulomb excitation experiment [8]. Blocking one of the resonant transitions removes the laser ionized component of the ion beam directed to an experiment, while preserving the contamination ionized by other mechanisms, thereby enabling the identification of signals related to the isotope of interest. The tellurium ionization scheme development was detailed in [9]. In order to be applied for standard radioactive ion beam production at ISOLDE, the efficiency of the ionization scheme had to be determined. The results of the efficiency measurement are presented in Section 3.3. 


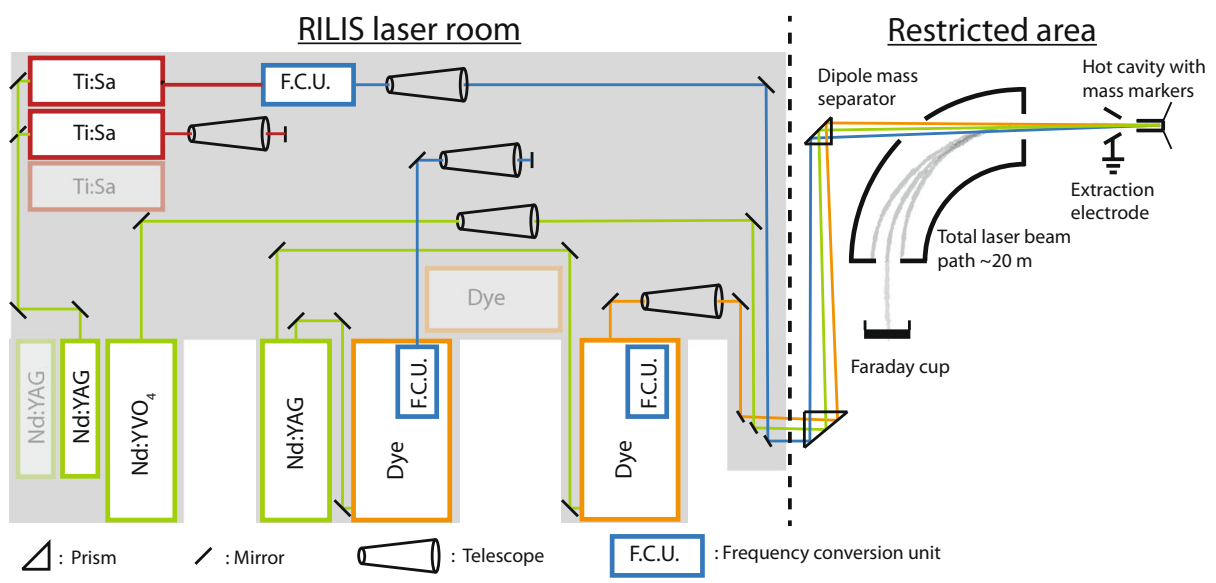

Fig. 1 Schematic of the experimental set-up used for the development and testing of ionization schemes of mercury and tellurium for the ISOLDE RILIS

\section{Experimental method}

The process of RILIS ionization scheme development at ISOLDE is outlined in [9] and [10]. Following the identification of potentially suitable atomic transitions based on literature data, spectroscopic studies and efficiency measurements are carried out. During standard ISOLDE operation for the production of radioactive isotopes, reaction products are produced by impinging $1.4 \mathrm{GeV}$ protons onto target material mounted inside an ISOLDE target units. The target material is heated depending on the material type, enabling the reaction products to diffuse through the target material and then effuse via a transfer line to the ion source. For RILIS operation and ionization scheme development, a hot cavity ion source (a tungsten or tantalum tube $34 \mathrm{~mm}$ in length with an internal diameter of $3 \mathrm{~mm}$ and heated to $\sim 2000^{\circ} \mathrm{C}$ ) is typically used. The ionization scheme development reported here took place at ISOLDE. A modified target unit was used, containing a hot cavity ion source with an independently heated refractory metal capillary attached to the rear, but no target for nuclear reactions. The capillary contained a stable sample "mass marker" of the element of interest. The modified target unit was mounted on a "front-end" connected to one of the dipole mass separators and optically to the ISOLDE RILIS laser system. This configuration for 'off-line' ionization scheme development, ensures the applicability of the results to "on-line" ISOLDE operation. The RILIS lasers are a combination of tunable dye and titanium:sapphire (Ti:Sa) lasers and non-tunable Nd:YAG and Nd:YVO 4 lasers, all pulsed at $10 \mathrm{kHz}$. The full laser parameters can be found in [2]. A schematic of the experimental set-up is presented in Fig. 1.

As depicted in Fig. 1, laser light from the RILIS lasers is focussed using telescopes located in the laser room and directed via an optical launch and transport system, through a window in the dipole separator magnet, to converge in an ion source cavity. During ionization scheme development, a mass marker containing the equivalent of $\sim 10000 \mathrm{nAh}$ of the element of interest is used (assuming their $100 \%$ conversion to singly charged ions), 
to ensure a sufficient supply of atoms during the spectroscopic investigations. The known resonant transitions are verified by directing the lasers to the ion source while heating the excess mass marker and detecting the ions on a Faraday cup inserted in the focal plane of the dipole mass separator. Following this, a tunable final step laser is introduced to search for autoionizing states or investigate Rydberg levels. While the final step laser is scanned, the ion current is measured with the Faraday cup and the laser wavelengths are measured with a HighFinesse/Ångstrom WS7 wavelength meter. The various experimental parameters are combined and recorded by a LabVIEW based data recording system [11]. Once an optimal scheme has been determined, a calibrated mass marker of typically between $1000 \mathrm{nAh}$ and $2000 \mathrm{nAh}$ is evaporated, while the ion current is measured with a Faraday cup. A Faraday cup measurement is recorded periodically during the evaporation of the sample, to enable the determination of the average recorded signal and thus the efficiency of the ionization scheme. During the tests reported here, a Faraday cup measurement was recorded every $10 \mathrm{~s}$ and $1 \mathrm{~s}$ for the mercury and tellurium efficiency measurements respectively. The final efficiency value is therefore a combined efficiency of the ionization, extraction and ion beam transport through the dipole mass separator. No attempt is made to correct for these factors as they are intrinsic to the system, thus a corrected value would not provide a realistic determination of the achievable efficiency of the entire machine.

The use of ISOLDE front-ends, mass separators and beam lines is limited due to the annual demand for physics, development or commissioning work. Consequently, only one efficiency measurement per element is typically possible. In the standard (target-transfer line-hot cavity) configuration used for on-line ISOLDE experiments, there is an additional laser-atom overlap region in the transfer line, increasing the efficiency of the ionization process [12]. The efficiency measured with the (mass marker-hot cavity) configuration, used for scheme development, could therefore be considered as a lower limit of ionization efficiency.

\section{Results and discussion}

\subsection{RILIS ionization scheme development for mercury}

The initial RILIS tests of the $\{254 \mathrm{~nm}|313 \mathrm{~nm}| 626 \mathrm{~nm}\}$ scheme took place prior to the upgrade to solid state pump lasers [1] and the addition of a complementary suite of Ti:Sa lasers [13]. Consequently, the ionization scheme was applied as suggested in [4], with the residual $626 \mathrm{~nm}$ laser light used for the final step transition (to what had been reported to be a favourable part of the ionization continuum). A full characterization of the scheme was possible in this work using the upgraded RILIS system. The first step $254 \mathrm{~nm}$ laser light was produced by $3 \omega$ frequency conversion of light from a Ti:Sa laser and the second step $313 \mathrm{~nm}$ light by $2 \omega$ frequency conversion of light produced by a Sirah Credo Dye laser, operated with an ethanol solution of DCM dye. The third step $626 \mathrm{~nm}$ light was produced by a second Sirah Credo Dye laser, also operated with an ethanol solution of DCM dye, enabling the wavelength of the final step to be scanned, while measuring the ion current. Scans of the third step revealed that the region of the ionization continuum around $87293 \mathrm{~cm}^{-1}$ was not found to be additionally favourable for ionization when accessed via $\{254 \mathrm{~nm}|313 \mathrm{~nm}| 626 \mathrm{~nm}\}$, with all three second steps tested exciting to $5 \mathrm{~d}^{10} 6 \mathrm{~s} 6 \mathrm{~d}{ }^{1} \mathrm{D}_{2}$, ${ }^{3} \mathrm{D}_{1},{ }^{3} \mathrm{D}_{2}$. Consequently, a comprehensive ionization scheme development programme was embarked upon. 


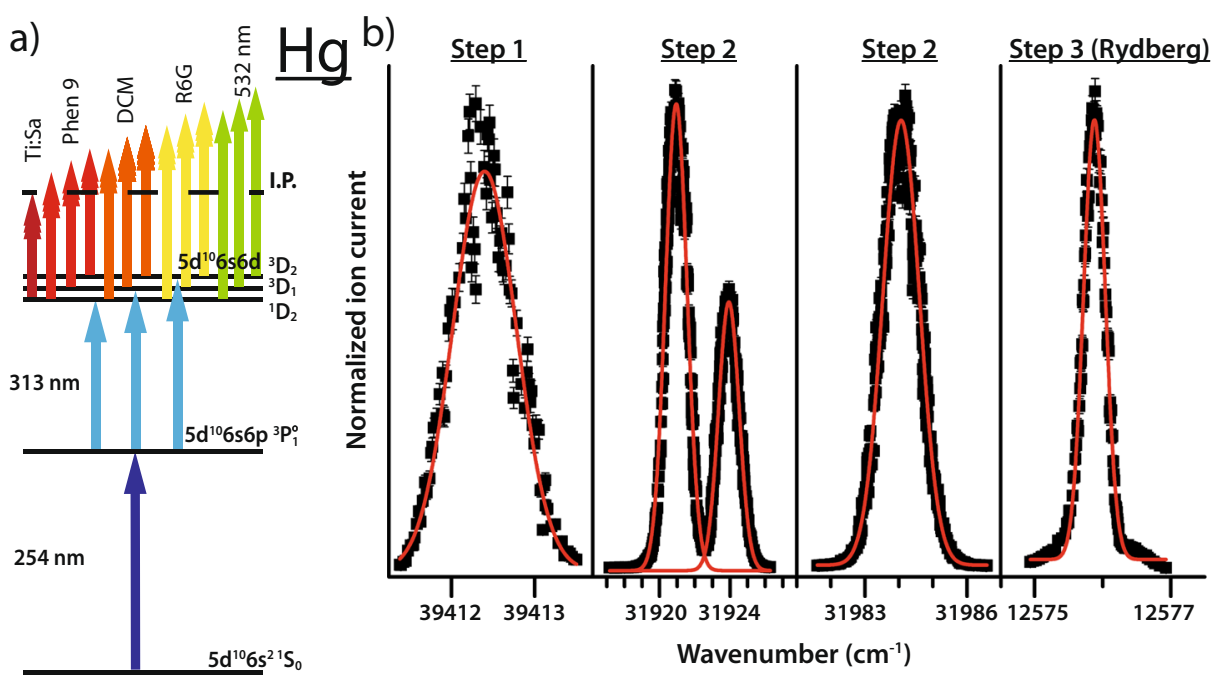

Fig. 2 a Schematic overview of the ionization scheme development for mercury. b Scans of the first step, three second steps and the optimal Rydberg resonance. The resonances were scanned while being applied as part of the the schemes depicted in (a)

Ionization schemes applying each of the three second step transitions were tested. The final step dye laser was scanned to probe the ionization continuum from each of them, while operated with ethanol solutions of either Rhodamine 6G, DCM or Phenoxazone 9 dyes. Rydberg levels were also investigated, exciting from the $5 \mathrm{~d}^{10} 6 \mathrm{~s} 6 \mathrm{~d}{ }^{1} \mathrm{D}_{2}$ state. Finally, the effect of the addition of a non-resonant transition ${ }^{(N R)}$ at $532 \mathrm{~nm}$ was tested, again exciting from each of the second steps, this is presented in Fig. 2a. The resonances corresponding to the first step, three second steps and the Rydberg level corresponding to the largest ion signal are presented in Fig. $2 \mathrm{~b}$.

The saturation of the second steps was verified. Saturation measurements of the first step were not fully conclusive, though saturation effects were observed with an estimated $15 \mathrm{~mW}$ of laser power delivered to the ion source. No autoionizing resonances were identified and the $88759.6 \mathrm{~cm}^{-1}$ autoionizing state reported in literature [14] was not observed. Based on the reported odd parity and $\mathbf{J}=1$, this state should have been accessible from each of the $5 d^{10} 6 s 6 d{ }^{1} D_{2},{ }^{3} D_{1},{ }^{3} D_{2}$ states. The laser parameters used for the scheme development and efficiency measurement are presented in Table 1 . The $\{254 \mathrm{~nm}|313.18 \mathrm{~nm}| 795 \mathrm{~nm}\}$ ionization scheme, ionizing via the Rydberg level shown in Fig. 2, was compared to the $\left\{254 \mathrm{~nm}|313.18 \mathrm{~nm}| 532 \mathrm{~nm}^{N R}\right\}$ scheme with a non-resonant final step to the ionization continuum using $532 \mathrm{~nm}$ light.

Ionization with the non-resonant final step exciting from the $5 \mathrm{p}^{3}\left({ }^{4} \mathrm{~S}^{\circ}\right) 7 \mathrm{p}{ }^{1} \mathrm{D}_{2}$ atomic state was determined to be four times more efficient than via the Rydberg level. This was achieved with an estimated $24 \mathrm{~W}$ of $532 \mathrm{~nm}$ laser light transmitted to the ion source from the $\mathrm{Nd}: \mathrm{YVO}_{4}$ Lumera Blaze laser [16], compared to an estimated $1.2 \mathrm{~W}$ of $795 \mathrm{~nm}$ laser light from a Ti:Sa laser. This discrepancy in the signal to power ratio can be understood as resulting from a higher cross-section of excitation to the Rydberg level. The 
Table 1 The schemes originated from the $5 \mathrm{~d}^{10} 6 \mathrm{~s}^{21} \mathrm{~S}_{0}$ atomic ground state. Spectroscopic information for known transitions is taken from [14]

\begin{tabular}{llll}
\hline $\begin{array}{l}\text { Transition } \\
\left(\mathrm{cm}^{-1}\right)\end{array}$ & Upper state config., term, J & $\begin{array}{l}\text { Air wavelength } \\
(\mathrm{nm})\end{array}$ & $\begin{array}{l}\text { laser power } \\
\mathrm{mW}\end{array}$ \\
\hline $0-39412.237^{*}$ & $5 \mathrm{~d}^{10} 6 \mathrm{~s} 6 \mathrm{p},{ }^{3} \mathrm{P}_{1}^{\circ}$ & 253.652 & 80 \\
$39412.237-71333.053^{*}$ & $5 \mathrm{~d}^{10} 6 \mathrm{~s} 6 \mathrm{~d},{ }^{1} \mathrm{D}_{2}$ & 313.184 & 120 \\
$39412.237-71336.005$ & $5 \mathrm{~d}^{10} 6 \mathrm{~s} 6 \mathrm{~d},{ }^{3} \mathrm{D}_{1}$ & 313.155 & 120 \\
$39412.237-71396.073$ & $5 \mathrm{~d}^{10} 6 \mathrm{~s} 6 \mathrm{~d},{ }^{3} \mathrm{D}_{2}$ & 312.567 & 120 \\
$71333.053-83908.95(4)$ & $\mathrm{Rydberg}$ & $794.955^{\dagger}(25)$ & 1200 \\
$71333.053-\sim 90121^{*}$ & - & 532 & 24000 \\
\hline
\end{tabular}

The given laser power is an estimation of the power delivered to the ion source. *Transitions applied for the efficiency measurement. ${ }^{\dagger}$ Air wavelength calculated using the equation of [15]

$\left\{254 \mathrm{~nm}|313.18 \mathrm{~nm}| 532 \mathrm{~nm}^{N R}\right\}$ ionization scheme was therefore determined to be the optimal one for application at the ISOLDE RILIS.

\subsection{RILIS ionization efficiency of mercury}

The $\left\{254 \mathrm{~nm}|313.18 \mathrm{~nm}| 532 \mathrm{~nm}^{N R}\right\}$ scheme was applied and the dipole mass separator was set to ${ }^{202} \mathrm{Hg}$, the isotope of mercury with the highest natural abundance (29.86 (26) \% [17]). The laser parameters used for the efficiency measurement are indicated with an $*$ in Table 1. A calibrated sample of $1336 \mathrm{nAh}$ of mercury $\left(\approx 400 \mathrm{nAh}\right.$ of $\left.{ }^{202} \mathrm{Hg}\right)$ was evaporated from the independently heated mass marker described in Section 2, while the ion current was measured with a Faraday cup inserted downstream of the dipole mass separator. The results are presented in Fig. 3, together with a schematic of the ionization scheme applied during the efficiency measurement.

An average signal of $13.2 \mathrm{nA}$ was recorded over a period of $1.88 \mathrm{~h}$, this corresponds to an efficiency of $6 \%$. This efficiency is a factor of 60 greater than the measured efficiency of the $\{254 \mathrm{~nm}|313.18 \mathrm{~nm}| 626 \mathrm{~nm}\}$ ionization scheme. During the previous efficiency measurement, the final ionizing transition was an estimated $1.3 \mathrm{~W}$ of $626 \mathrm{~nm}$ laser light delivered to the ion source. Considering the fact that the ionizing transitions are accessing different areas of the ionization continuum and the efficiency was only measured once for each scheme, this is within expectations.

Following these results, the new RILIS scheme was applied using the anode cavity of a VADIS (ISOLDE's FEBIAD type) ion source as the laser atom interaction region, described in [18]. The extracted mercury ion currents when using either the arc discharge ionization process or the element selective RILIS ionization process were found to be equivalent, suggesting a comparative efficiency under on-line conditions. The new ionization scheme was then successfully applied for an in-source resonance ionization spectroscopy of mercury experiment, extending the hyperfine structure measurements four isotopes further towards the neutron dripline to ${ }^{177} \mathrm{Hg}$ and two isotopes further in the neutron rich direction up to ${ }^{208} \mathrm{Hg}$ [19].

\subsection{RILIS ionization efficiency of tellurium}

The new RILIS ionization scheme for tellurium of $\{214 \mathrm{~nm}|573 \mathrm{~nm}| 901 \mathrm{~nm}\}$ (described in [9]) was applied and the dipole mass separator was set to ${ }^{130} \mathrm{Te}$, the isotope of tellurium 

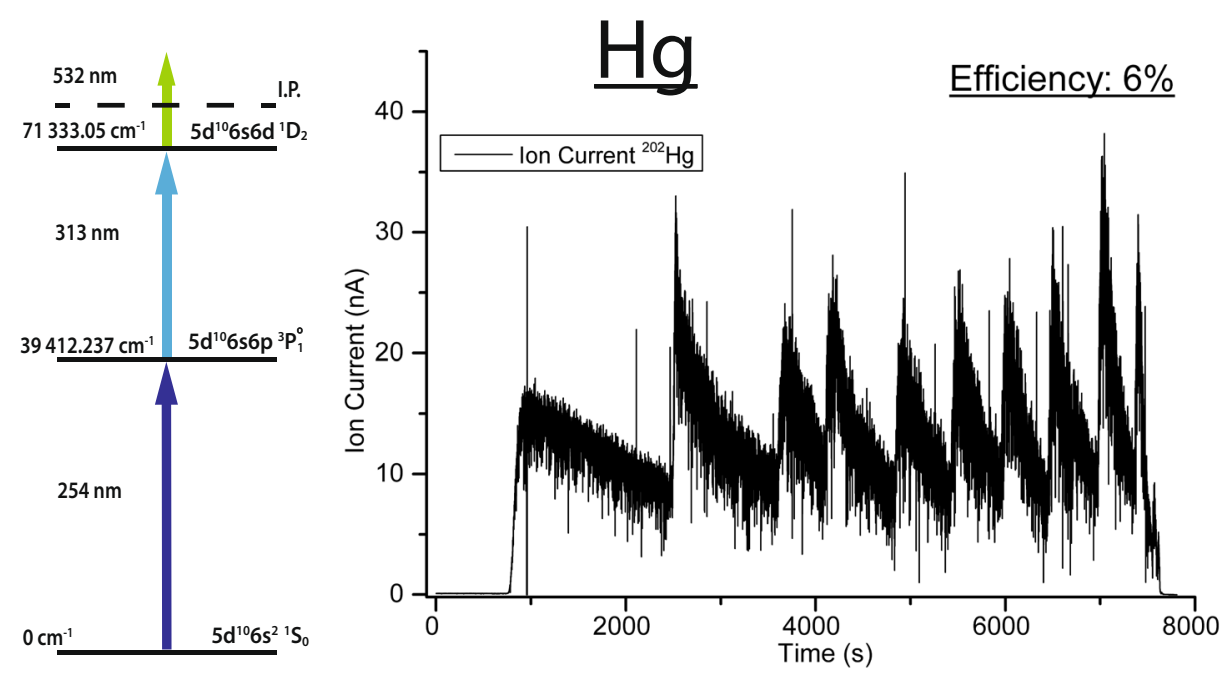

Fig. 3 The measured ion current over the period of the efficiency measurement, together with a schematic of the ionization scheme that was applied. Additional laser parameters are presented in Table 1

Table 2 The resonant transitions for the scheme originating from the $5 \mathrm{p}^{4}{ }^{3} \mathrm{P}_{2}$ atomic ground state applied during the tellurium ionization scheme efficiency measurement

\begin{tabular}{llll}
\hline $\begin{array}{l}\text { Transition } \\
\left(\mathrm{cm}^{-1}\right)\end{array}$ & $\begin{array}{l}\text { Upper state config., term, J } \\
\left(\mathrm{cm}^{-1}\right)\end{array}$ & $\begin{array}{l}\text { Air wavelength } \\
(\mathrm{nm})\end{array}$ & $\begin{array}{l}\text { laser power } \\
(\mathrm{mW})\end{array}$ \\
\hline $0-46652.738$ & $5 \mathrm{p}^{3}\left({ }^{4} \mathrm{~S}^{\circ}\right) 6 \mathrm{~s},{ }^{3} \mathrm{~S}^{\circ}, 1$ & 214.281 & 13 \\
$46652.738-64088.997$ & $5 \mathrm{p}^{3}\left({ }^{4} \mathrm{~S}^{\circ}\right) 7 \mathrm{p},{ }^{3} \mathrm{P}, 1$ & 573.360 & 4000 \\
$64088.997-75181.41(20)$ & - & $901.270(17)$ & 1300 \\
\hline
\end{tabular}

The given laser power is an estimation of the power delivered to the ion source. Spectroscopic information taken from [9]

with the highest natural abundance (34.08 (62) \% [17]). The laser parameters during the tellurium efficiency measurements are presented in Table 2.

A calibrated sample of $1000 \mathrm{nAh}$ of tellurium $\left(\approx 340 \mathrm{nAh}\right.$ of $\left.{ }^{130} \mathrm{Te}\right)$ was evaporated from the independently heated mass marker described in Section 2, while the ion current was measured with a Faraday cup inserted downstream of the dipole mass separator. The results are presented in Fig. 4 together with a schematic of the ionization scheme that was applied during the measurements.

A data acquisition malfunction resulted in the loss of the Faraday cup measurements towards the end of the measurement. However, sufficient data was recorded to enable a reliable extrapolation of the ion current to cover the missing data points using an exponential decay function. This is depicted in Fig. 4 as a dashed (red) line. The accuracy of the extrapolation was aided by the possibility to measure the ion current once the correct functioning of the data acquisition system had been recovered, providing an end point for the exponential extrapolation. The increase in the ion signal at the end of the efficiency measurement corresponds to a final heating of the mass marker capillary. Due to time constraints, it was 


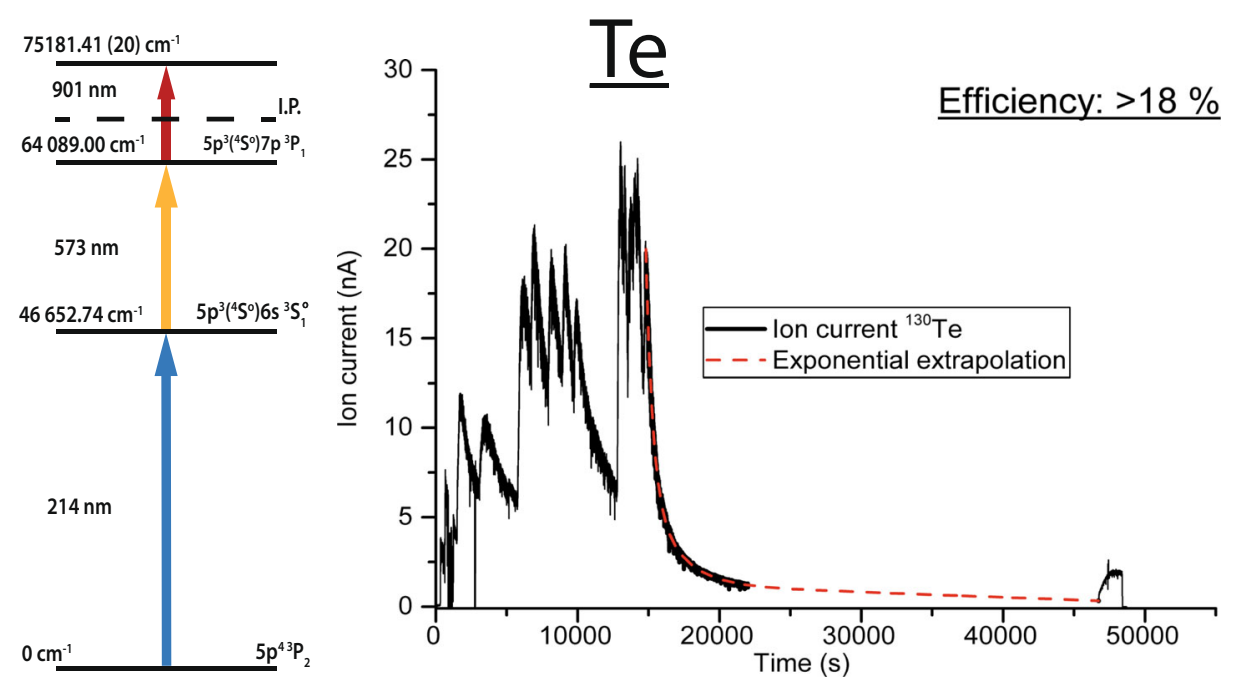

Fig. 4 The measured ion current over the period of the efficiency measurement, together with a schematic of the ionization scheme that was applied. Additional laser parameters are presented in Table 2

necessary to stop the measurement while the ion rate was stable and equivalent to $0.6 \%$ of efficiency/hour. This indicates there was a significant amount of tellurium remaining in the system. As there was not sufficient data to reliably extrapolate the decay of the ion rate following the final heating, only the data shown in Fig. 4 was included in the evaluation of the efficiency. An average signal of $4.8 \mathrm{nA}$ was recorded over a period of $12.98 \mathrm{~h}$, this corresponds to an efficiency of $18 \%$ which is considered to be a lower limit for the efficiency of the scheme.

\section{Conclusions}

An optimal RILIS ionization scheme of $\left\{254 \mathrm{~nm}|313.18 \mathrm{~nm}| 532 \mathrm{~nm}^{N R}\right\}$ was determined for mercury. A scheme of $\{254 \mathrm{~nm}|313.18 \mathrm{~nm}| 795 \mathrm{~nm}\}$, ionizing via a Rydberg level, corresponded to a factor of four lower ion current at typical laser power levels available at the ISOLDE RILIS for these wavelengths. No autoionizing resonances were observed, including the autoionizing state reported in literature at $88759.6 \mathrm{~cm}^{-1}$. The efficiency of the new RILIS ionization scheme for mercury of $\left\{254 \mathrm{~nm}|313.18 \mathrm{~nm}| 532 \mathrm{~nm}^{N R}\right\}$ was determined to be $6 \%$, a factor of 60 higher than the previously measured efficiency of $0.1 \%$ with a scheme of $\{254 \mathrm{~nm}|313 \mathrm{~nm}| 626 \mathrm{~nm}\}$. The efficiency of the new RILIS ionization scheme for tellurium $(\{214 \mathrm{~nm}|573 \mathrm{~nm}| 901 \mathrm{~nm}\}$ reported in [9]) was determined to be $>18 \%$, with a significant amount of tellurium remaining in the system at the end of the measurement. The mercury scheme has already been applied at ISOLDE for a successful in-source resonance ionization spectroscopy experiment. The scheme was demonstrated to have an ionization efficiency equivalent to that of the ISOLDE VADIS ion source under standard on-line conditions [18]. The application of the new mercury scheme has been requested for a number of future ISOLDE experiments, due to the selective method of ionization offered by the RILIS. 
Acknowledgments This project has received funding through the European Union's Seventh Framework Programme for Research, Technological Development and demonstration under Grant Agreements 262010 (ENSAR), 267194 (COFUND), and 289191 (LA ${ }^{3}$ NET).

Open Access This article is distributed under the terms of the Creative Commons Attribution 4.0 International License (http://creativecommons.org/licenses/by/4.0/), which permits unrestricted use, distribution, and reproduction in any medium, provided you give appropriate credit to the original author(s) and the source, provide a link to the Creative Commons license, and indicate if changes were made.

\section{References}

1. Fedosseev, V., Berg, L., Fedorov, D., Fink, D., Launila, O., Losito, R., Marsh, B., Rossel, R., Rothe, S., Seliverstov, M., Sjodin, A., Wendt, K.: Upgrade of the resonance ionization laser ion source at ISOLDE on-line isotope separation facility: New lasers and new ion beams. Rev. Sci. Instrum. 83(2), 02 A903 (2012)

2. Rothe, S., Day Goodacre, T., Fedorov, D., Fedosseev, V., Marsh, B., Molkanov, P., Rossel, R., Seliverstov, M., Veinhard, M., Wendt, K.: Laser ion beam production at CERN-ISOLDE: New features - More possibilities. Nuc. Instrum. Methods Phys. Res. Sec. B: Beam Inter. Mater. Atoms 376, 91-96 (2016)

3. Kugler, E.: The ISOLDE facility. Hyp. Inter. 129(1/4), 23-42 (2000)

4. Podshivalov, A., Matveev, O., Smith, B., Winefordner, J.: A novel and efficient excitation- and ionization-scheme for laser resonance ionization of mercury. Spectrochim. Acta Part B: Atom. Spectros. 54(13), 1793-1799 (1999)

5. Fedosseev, V., Berg, L., Lebas, N., Launila, O., Lindroos, M., Losito, R., Marsh, B., Ȯsterdahl, F., Pauchard, T., Transtromer, G., Vannesjö, J.: ISOLDE RILIS: New beams, new facilities. Nuc. Instrum. Methods Phys. Res. Sect. B: Beam Inter. Mater. Atoms 266(19-20), 4378-4382 (2008)

6. Sundell, S., Ravn, H.: Ion source with combined cathode and transfer line heating. Nuc. Instrum. Methods Phys. Res. Sec. B: Beam Inter. Mater. Atoms 70(1-4), 160-164 (1992)

7. Gaffney, L., Day Goodacre, T., Andreyev, A., Seliverstov, M., Althubiti, N., Andel, B., Antalic, S., Atanasov, D., Barzakh, A., Billowes, J., Blaum, K., Cocolios, T., Cubiss, J., Farooq-Smith, G., Fedosseev, V., Fedorov, D., Ferrer, R., Flanagan, K., Ghys, L., Gottberg, A., Granados, C., Herfurth, F., Huyse, M., Jenkins, D., Kisler, D., Kreim, S., Kron, T., Kudryavtsev, Y., Lunney, D., Lynch, K., Manea, V., Marsh, B., Mendonca, T., Molkanov, P., Neidherr, D., Raabe, R., Raeder, S., Ramos, J., Rapisarda, E., Rosenbusch, M., Rossel, R., Rothe, S., Schweikhard, L., Sels, S., Stora, T., Tsekhanovich, I., Van Beveren, C., Van Duppen, P., Veinhard, M., Wadsworth, R., Wienholtz, F., Welker, A., Wendt, K., Wilson, G., Witkins, S., Wolf, R., Zuber, K.: In-source laser spectroscopy of mercury isotopes. CERN INTC (P-424) (2014)

8. Ahn, T., Al-Azri, H., Bloch, T., Butler, P., Bree, N., Bảck, T., Bỏnig, S., Cederkảll, J., Cederwall, B., Darby, I., Diriken, J., O’Donnell, D., Fahlander, C., Gaffney, L., Grahn, T., Hadinia, B., Huyse, M., Jenkins, D., Johnson, A., Joshi, P., Joss, D., Julin, R., Kröll, T., Leske, J., Nara Singh, B., Nicholls, A., Page, R., Pakarinen, J., Paul, E., Pietralla, N., Rahkila, P., Rapisarda, E., Sandzelius, M., Scheck, M., Simpson, J., Smith, J., Wadsworth, R., Van Duppen, P., Voulot, D., Wenander, F., Werner, V.: Coulomb excitation of ${ }^{116} \mathrm{Te}$ and ${ }^{118} \mathrm{Te}$ : A study of collectivity above the $Z=50$ shell gap. CERN-INTC, 11 (2011)

9. Day Goodacre, T., Fedorov, D., Fedosseev, V., Forster, L., Marsh, B., Rossel, R., Rothe, S., Veinhard, M.: Laser resonance ionization scheme development for tellurium and germanium at the dual Ti:Sa-Dye ISOLDE RILIS. Nuc. Instrum. Methods Phys. Res. Sec. A: Accel. Spectrom. Detect. Assoc. Equip. 830, 510-514 (2016)

10. Fedosseev, V., Marsh, B., Fedorov, D., Köster, U., Tengborn, E.: Ionization Scheme Development at the ISOLDE RILIS. Hyp. Inter. 162(1-4), 15-27 (2006)

11. Rossel, R., Fedosseev, V., Marsh, B., Richter, D., Rothe, S., Wendt, K.: Data acquisition, remote control and equipment monitoring for ISOLDE RILIS. Nuc. Instrum. Methods Phys. Res. Sec. B: Beam Inter. Mater. Atoms 317, 557-560 (2013)

12. Liu, Y., Baktash, C., Beene, J., Havener, C., Krause, H., Schultz, D., Stracener, D., Vane, C., Geppert, C., Kessler, T., Wies, K., Wendt, K.: Time profiles of ions produced in a hot-cavity resonant ionization laser ion source. Nuc. Instrum. Methods Phys. Res. Sec. B: Beam Inter. Mater. Atoms 269(23), 27712780 (2011) 
13. Rothe, S., Marsh, B., Mattolat, C., Fedosseev, V., Wendt, K.: A complementary laser system for ISOLDE RILIS. J. Phys. Conf. Ser. 312(5), 052020 (2011)

14. Kramida, A., Ralchenko, Y., Reader, J.: The NIST ASD Team: NIST Atomic Spectra Database (ver 5.2). [Online, accessed: 22/07/2016] (2015)

15. Birch, K., Downs, M.: An updated Edlén equation for the refractive index of air. Metrologia 30(3), 155162 (1993)

16. Marsh, B., Day Goodacre, T., Fink, D., Rothe, S., Seliverstov, M., Imai, N., Sjodin, M., Rossel, R.: Suitability test of a high beam quality Nd:YVO4 industrial laser for the ISOLDE RILIS installation. Technical report, CERN, Geneva (2013)

17. de Laeter, J., Bòhlke, J., De Bièvre, P., Hidaka, H., Peiser, H., Rosman, K., Taylor, P.: Atomic weights of the elements. Review 2000 (IUPAC Technical Report). Pure Appl. Chem., 75(6) (2003)

18. Day Goodacre, T., Billowes, J., Catherall, R., Cocolios, T., Crepieux, B., Fedorov, D., Fedosseev, V., Gaffney, L., Giles, T., Gottberg, A., Lynch, K., Marsh, B., Mendonċa, T., Ramos, J., Rossel, R., Rothe, S., Sels, S., Sotty, C., Stora, T., Van Beveren, C., Veinhard, M.: Blurring the boundaries between ion sources: The application of the RILIS inside a FEBIAD type ion source at ISOLDE. Nuc. Instrum. Methods Phys. Res. Sec. B: Beam Inter. Mater. Atoms 376, 39-45 (2016)

19. Day Goodacre, T.: Developments of the ISOLDE-RILIS for radioactive ion beam production and the results of their application in the study of exotic mercury isotopes. $\mathrm{PhD}$ thesis, The University of Manchester (2017) 